\title{
Effective Trace Acquirement during Product Information Diffusion and Application
}

\author{
Xuehua Sun*, Jianjun Wang, Guozhang Xu, Hongwei Gao, Liping Ma \\ Computer Center, Hebei University of Economics and Business, Shijiazhuang, China \\ Email: *sunxuehua795@126.com,wangjj65@126.com,18132012380@163.com,hongwgao@163.com,mLPbox@126.com
}

How to cite this paper: Sun, X.H., Wang, J.J., Xu, G.Z., Gao, H.W. and Ma, L.P. (2020) Effective Trace Acquirement during Product Information Diffusion and Application. Intelligent Information Management, 12, 105-120.

https://doi.org/10.4236/iim.2020.123008

Received: March 28, 2020

Accepted: May 3, 2020

Published: May 6, 2020

Copyright $\odot 2020$ by author(s) and Scientific Research Publishing Inc. This work is licensed under the Creative Commons Attribution International License (CC BY 4.0).

http://creativecommons.org/licenses/by/4.0/

\begin{abstract}
Information dissemination has become part of people's daily communication and there is great interest for both academic and industrial communities. Most previous studies have focused on the strategy and mechanisms. The methods controlling the process of information diffusion have rarely been studied. Thus, previous studies have failed to effectively mine the value of product information diffusion on social networks. In this study, based on the information diffusion product in consumer self-organized social networks, the control of the product information diffusion process was explored. The node identification principle of the QR code sender designed in this study and the linked list that associated information with specific nodes allowed the acquisition of effective traces in long-chain transmission from the information source to the value nodes, and solved user information disclosure during the transmission process. This method was applied to the tracing system of defective vehicles, achieving accurate recall of defective vehicles.
\end{abstract}

\section{Keywords}

Product Information Diffusion, Effective Trace Acquirement, Technical Process Reasoning, QR Code Sender

\section{Introduction}

WeChat, social media websites, and mobile intelligent terminals, as consumer-led media and technology, have developed rapidly. Consumers not only produce a great amount of data online but also spontaneously create their own marketing networks. This blurs the boundaries between enterprises and consumers. As content providers and information publishers, consumers have built their own media networks; however, enterprises publish abundant product information through various forms of social media, such as network platforms and 
intelligent media terminals, to attract the attention of potential users. Incentives have been offered to encourage consumers to share product information on their self-organized social media. Therefore, consumers are transformed from people who simply browse product information and purchase products into enterprise collaborators and product promoters. Such partnerships can benefit both consumers and enterprises.

Consumers form circles of friends on social media. Consumers do not always know how to make the right choices when faced with massive amounts of data and media advertising. Therefore, they prefer to receive recommendations from friends that they trust in their communication circles (Figure 1).

There are currently two avenues of information transmission; namely, the rumor diffusion model that was developed based on the epidemic model and information cascade. The classic epidemic disease model, Susceptible-Infectious-Recovered (SIR), proposed by Anderson in 1991, reference [1] [2], has been widely employed, especially for the spread of rumors. Scholars have improved the SIR model and the improved models are summarized in Table 1. The classic rumor-spread model is the Daley and Kendal (DK) model [3] [4] [5], which suggests that rumor spread is similar to the spread of infectious diseases. People are divided into three categories in the DK model: the ignorant, the spreader, and the terminator. In the SSIC model [6], the spread of rumors on super networks can be effectively hampered [7] by: 1) identifying rumors and isolating them, and 2) improving the extent of rumors, which allows the public to know more about them, to weaken their spread. The SEIR model [8] considers the ambiguity and attractiveness of the rumor content. Mean-field equations are used to characterize the dynamics in the SEIR model considering homogeneous and heterogeneous networks. Rumors spread faster in the BA network in the SEIR model than in the WS network, while the diffusion scales of rumors spread are exactly the opposite in the two networks. Mean-field equations are used to describe the dynamics of rumor models to consider the characteristics of rumors spreading and analyze the key events in complex networks. A novel SIR model [9] was

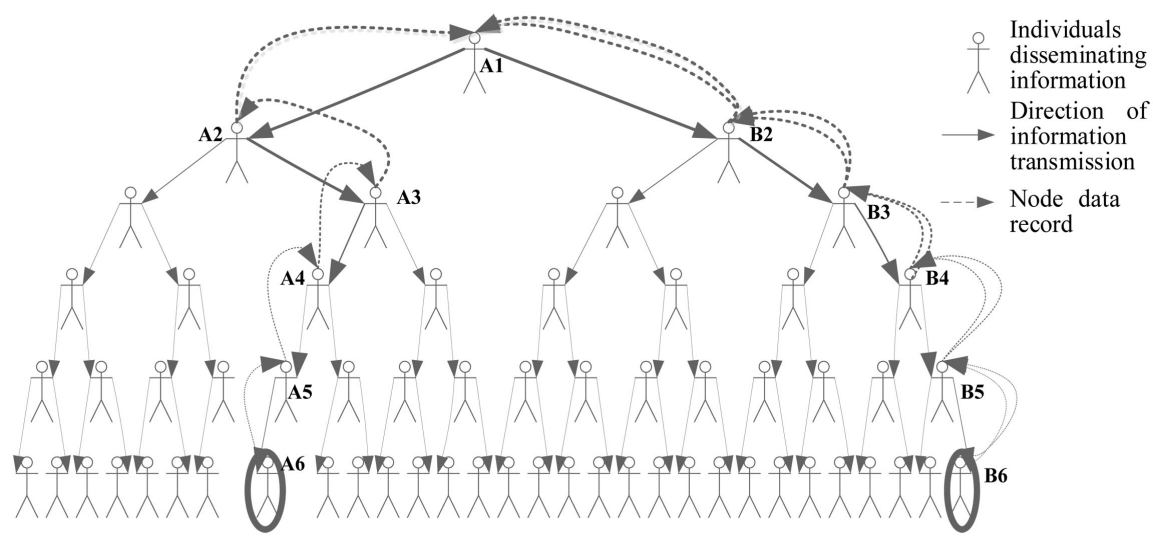

Figure 1. Product value creation and information sharing in networks self-organized by consumers. 
Table 1. Summary of improved rumor models.

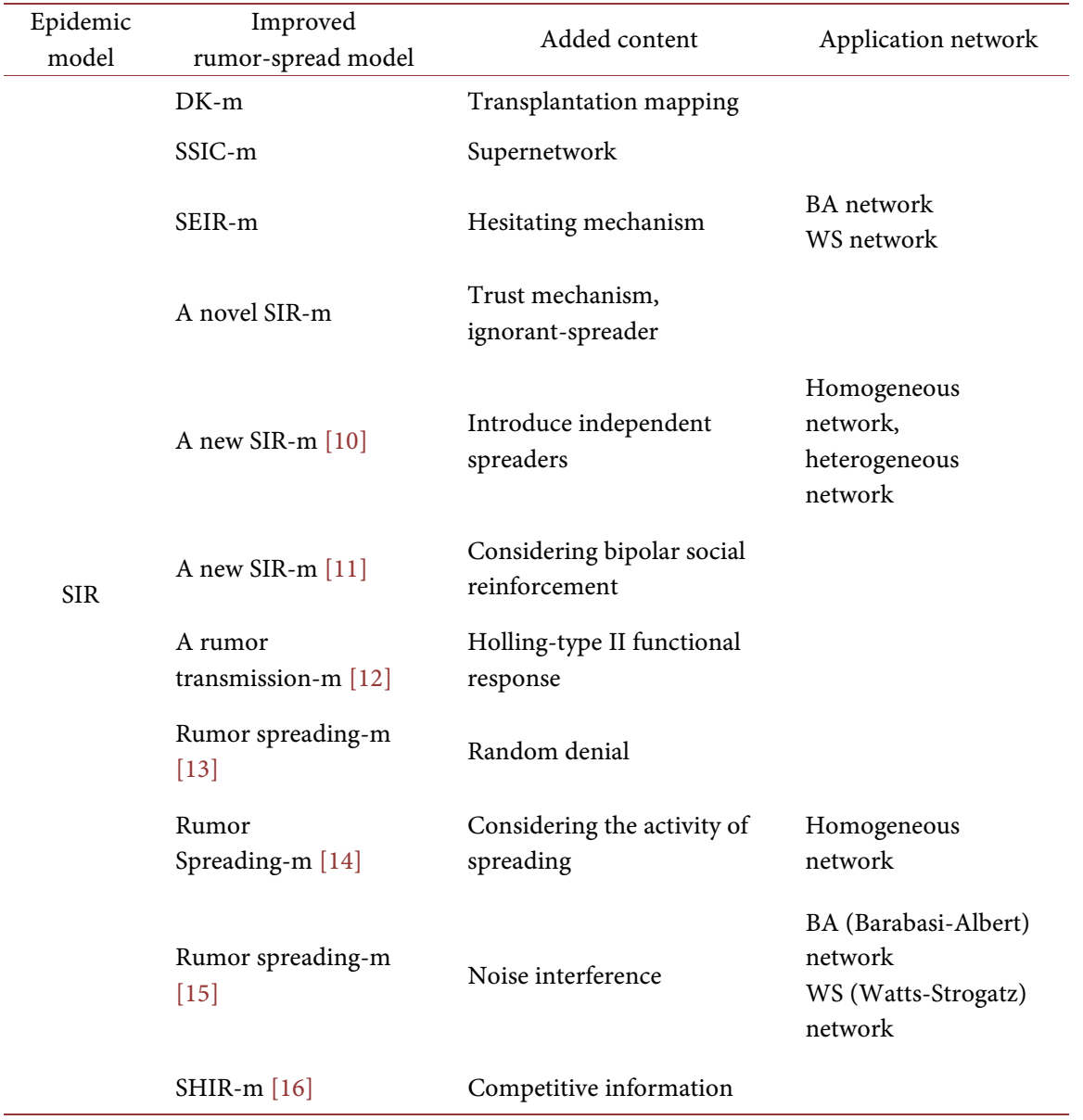

applied to heterogeneous/homogeneous networks. The results showed that rumors spread faster in homogeneous networks compared to heterogeneous networks. The diffusion scales of rumor spread in the two networks, however, were exactly the opposite, as shown in Table 1.

In general, understanding the spread of rumors has been improved using the SIR model, mainly in two aspects: 1) constructing network structures based on different attributes, and 2) increasing the attributes of the study objects.

The improved rumor spread model and the epidemic disease model differ from the information diffusion model in that networks are self-organized by consumers in two ways. First, information is diffused in various ways. Node infection is forced and spontaneous in the epidemic disease model, while information diffusion in a consumer network is optional and voluntary. Second, the targets vary. Scholars assess rumor models to disturb and suppress rumor spread in hopes of minimizing its impact. Studies on product information diffusion in consumer networks encourage consumers' forwarding behaviors and maximize their effects. Therefore, the epidemic model as well as the improved rumor model, which is based on the epidemic model, cannot abstractly represent information dissemination processes in consumer networks. 
Another main mode of information diffusion is information cascade. The most representative information diffusion models are discussed below. Reference [17] proposed the general cascade (GC) model and the general threshold (GT) model, which are generalizations of the independent cascade (IC) model and the linear threshold (LT) model, and the two models are equivalent. In the GC model, the probability of node diffusion was determined by the historical activation record, while this probability was set as a constant in the IC model. In the GT model, the threshold function determining whether a node was activated was the function of the weight of activated nodes; however, in the LT model, it was the sum of the weight of all the activated nodes. In the IC and LT models, each activated node attempted to influence nodes that were not activated; and in the voter model [18], each node had two options, and two types of information competed for activating more nodes.

In addition to the traditional information diffusion models, as mentioned above, there are also many expansion models based on these models. The IC model with negative opinions (IC-N) [19] also considers the diffusion of negative information. In the IC-N model, successful activation of silent nodes by a positive activated node will lead to the spread of positive and negative information simultaneously, but successful activation of silent nodes by a negative activated node will lead to the diffusion of only negative information. The competitive LT model (CLT) model [20] extended the LT model and considered two types of competing information in the network. In the CLT model, the seed nodes are activated and attached to one of the two competing information to be spread; when activating silent nodes, the seed nodes attempt to persuade the silent nodes to accept the information they supported. The Signed Voter model [21] is an extension of the Voter Model; in this model, when the two nodes on one edge are friends, one node successfully activates the other node and attempts to persuade it to accept the information he or she supports. The IC-N model and the CLT model produce a similar negative impact when successfully activating silent nodes, which makes the other node hold the opposite view.

In the above studies, a default hypothesis was commonly adopted, which was: the consumer spontaneous information diffusion cannot be controlled. Therefore, even if previous studies had realized that blindly launching advertising in social media was inefficient, they would have been able to do nothing but promote information diffusion through seeking and developing influential seed nodes. Once the seed nodes activate the forwarding behavior and start self-organized diffusion among consumers, the follow-up forwarding behavior will be invisible to the external diffusion network; and the enterprise cannot be sure whether the purchasing behavior provoked is driven by the selected seed node or not. Thus, the problems studied in the past are only about macroscopic strategic research or microscopic seed node identification [22] [23] [24] [25] [26].

Therefore, all the information diffusion models currently available are "black 
box models" that cannot control, trace, and record the information transmission process. The node identification principle of the QR code sender designed in this study and the linked list that associated information with specific nodes realized the acquisition of effective traces in long-chain transmission from the information source to the value nodes. The coding system for public and private codes solved user information disclosure during information diffusion. The above method has not only controlled the product information diffusion process, but can also be applied to the technical reasoning of defective products to achieve accurate recall of defective vehicles.

The logic structure of the study is as follows: In the second part, the method to obtain effective traces from the value node to the information source was explored. In the third part, the technical process reasoning of defective products was studied, including the tracing system of defective products and technical process reasoning of defective products based on the method to obtain effective trace. The conclusions are provided in the fourth part.

\section{Effective Trace Acquirement from Value Node to Information Source}

This section aims to obtain the effective trace from a given value node $z$ to the information source, thereby achieving control of the product information diffusion process. However, there should be a prerequisite; that is, to identify and record the nodes that diffuse information to obtain the network where information about one specific product is diffused.

The development of QR code technology enabled us to realize the above objectives. Consider the following scenario: When a consumer shops in the mall and sees something that his friend wanted to buy, he sends the QR code of this product to his friend who will buy this product if his demand happens to be satisfied after seeing the information linked to this code; or, if through a quantity of nodes, the information is finally sent to the person who needs this product, then the above process is the product information diffusion in the consumer self-organized network, with QR code as the carrier.

To obtain the above product information diffusion network, it is necessary to record and identify the nodes that diffuse the information and associate relevant information to those nodes, which is based on the QR code technology, as shown in Figure 2. In the process of information diffusion, only the product information is visible and the identification code linked with personal information is only visible to the code sender. On the premise that the transmission nodes agree to identify the personal information, when the enterprise wants to find out the contribution made by consumers, the personal information can be provided for relevant enterprises so that they can give awards to the transmission nodes that have created value so as to encourage their behavior of diffusing relevant product information in social networks.

Before a consumer forwards the information of a product, the two parts in 


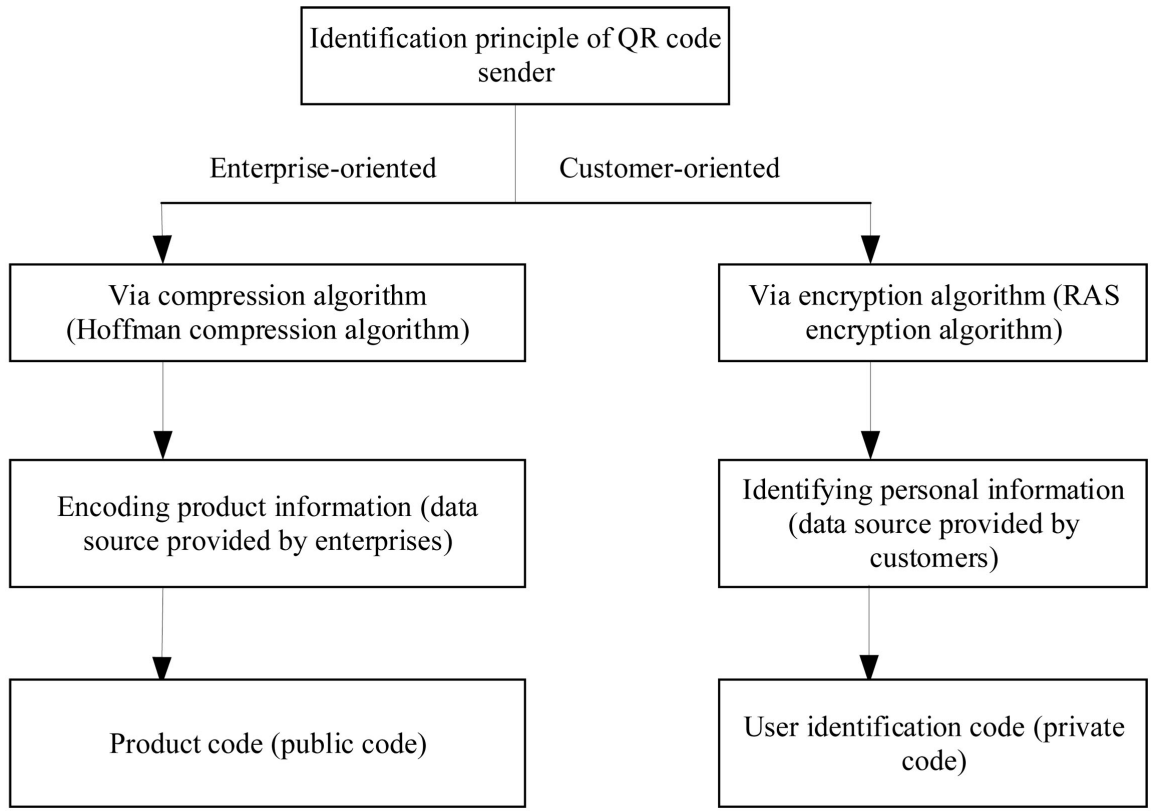

Figure 2. Node identification principle of the QR code sender.

Figure 2 are completely independent, and only when the consumer scans the information of a product, the two are associated, that is, the scanned QR code is attached with the consumer's personal information identification code, which is then entered into the database. If this consumer forwards the QR code to his friend, the QR code sent to his friend also contains the identification code of the forwarder in addition to the product information, but it is invisible to his friend who can only see the product information. In this way, user information during product information diffusion is protected, so that the disclosure of personal information can be avoided to the largest extent and consumers can diffuse product information without the worries behind it. The mechanism showing how the above process is carried out is shown in Figure 3.

Here, it is assumed that this consumer is willing to provide the only information that can reveal his personal identity to the QR code sender (e.g., WeChat ID, mobile phone quantity, QQ quantity, or an account registered on a social networking site etc.) so as to obtain the identification code (private) generated by this QR sender for this consumer, and the sender promises to keep the personal information confidential. If the user has entered his personal information and obtained the identification code, the system can automatically identify this user and attach his identification code to the product information if the consumer scans the QR code using the mobile APP developed by the QR sender.

If the consumer has not yet received the personal identification code from the QR code sender and scans the QR code using the APP, the system will pop up a page asking if it is allowed to generate the consumer's personal identification code to record the cumulative value contribution made by this consumer during product information diffusion and to be used in follow-up awards. It is assumed 


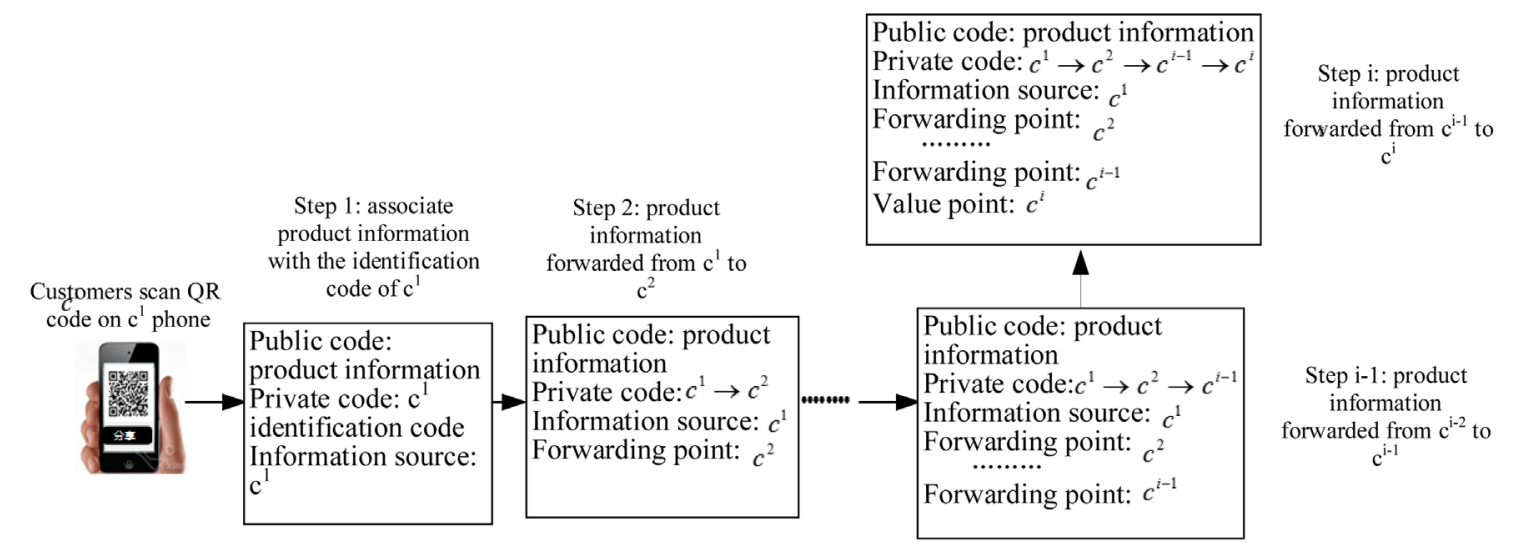

product

information

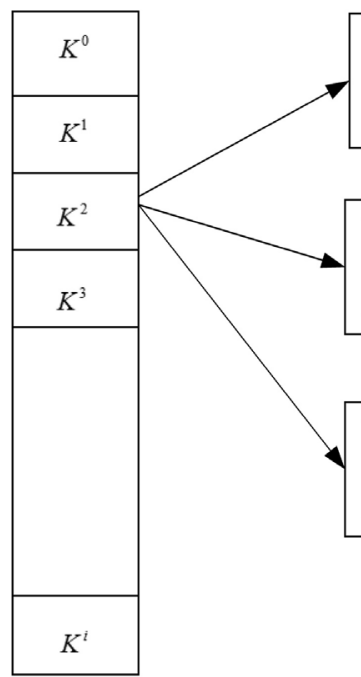

(a) nformation source: $c^{1}$ point:

rwarding point: $c^{i-1}$

ed from $c^{1}$ to

tep i-1: product

formation $\mathrm{c}^{\mathrm{i}-1}$

Figure 3. (a) Consumer information passing node identification mechanism; (b) The association of information and nodes in a linked list. Note: A linked list was used to save product information, identification nodes and the association between the two, and it was assumed that the probability of information forwarding between consumers is estimable. (b) The association method of the linked list was simulated with products of $K^{2}$ type as the case.

here that all consumers discussed in this study are willing to accept the invitation from the QR code sender to generate their personal identification codes.

If the node is an information source, a new linked list will be established under the directory of the product diffused by this node. If the information received by this node is from other consumers, the formation will be included in the existing linked list according to the product information and information source identification code.

When a consumer scans the QR code linked with certain product information, this code does not contain the identification codes of other users, and this consumer forwards the product information to his friend, then the consumer is an information source that diffuses information about this product.

To obtain the effective trace from the value node (the node generating purchasing) to the information source node, the information source corresponding 
to the value node should be found in the system's linked list structure according to the product-consumer catalogue. There may be more than one effective trace, for there may be more than one source of information that initiates the long chain spread to the value node $z$.

\section{Reasoning of the Technical Process of Defective Products}

The method to control product information diffusion; that is, the approach to obtain an effective trace was applied in technical process reasoning of defective products. According to the module production in the auto industry, the method to trace defective vehicles was designed. The product supply chain tracing model was constructed based on the parts batch relation and the order information system. In addition, the above QR code identification principle and the method for tracking effective trace were used to achieve the goals of detection of defective products, tracing to the cause, detection of defective products in the same batch, and accurate recall of defective products.

\subsection{Tracing System of Defective Products}

The recall of defective vehicles includes active recall and commanded recall. The tracing process is the same in both cases: Defective parts detected first, and then internal and external tracing is performed. Defective vehicles are caused by two major aspects; namely design and manufacturing. Design-related defective vehicles are often recalled based on the production time, while vehicles with defects caused during the production process are usually recalled per the production batch. Part suppliers and assembly unit suppliers are the information carriers in internal tracing. The information carriers in external tracing are vehicle manufacturers, dealers and client bases. The scope and quantity of defective products are confirmed according to the batch and quantity of defective parts determined by the internal tracing.

\subsubsection{Internal Tracing System}

For an auto production chain, the internal tracing system can be divided into three steps, as shown in Figure 4.

1) It is completed in the $4 \mathrm{~S}$ store or auto maintenance department where the technical personnel identify the defective parts, confirm the identification code and purchase batch of the defective parts according to the batch list, and trace the supplier $\mathrm{X}$ that have produced those parts.

2) The supplier $X$ inquires about the designer of the defective parts, processing/testing process of the parts and the workers who finished this process according to the identification code and production data of the parts. The batch and quantity of the designer, operator or defective products are determined to minimize the quantity of defective products.

The specific processes are: 1) to determine the link where the defects were caused. Causes of the defects are confirmed according to the process_id, operator_id_date, designer_id_date in information about processing of the defective 


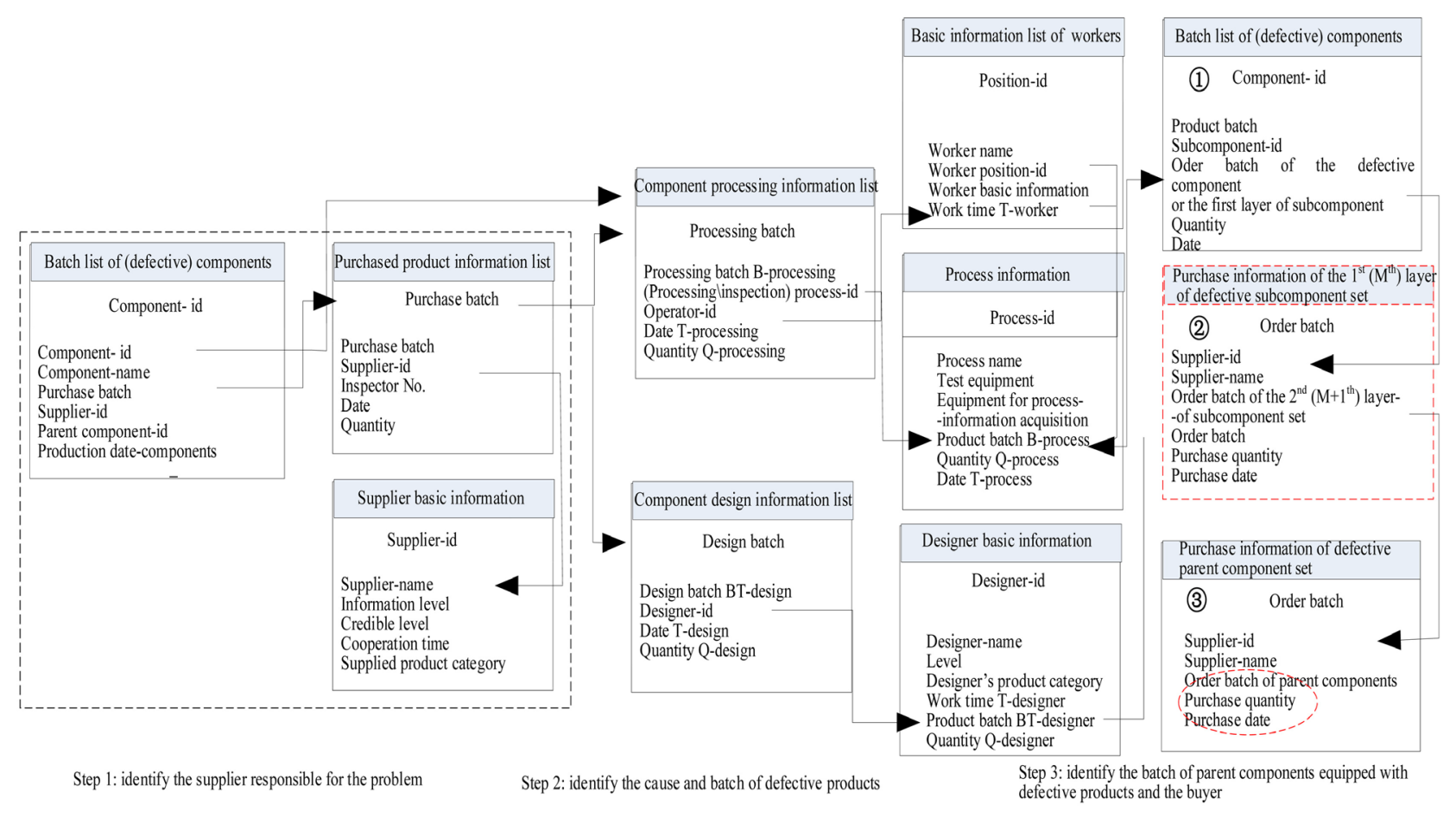

Figure 4. Internal tracing system.

parts: a. improper operation of the workers, b. problems that occurred in one specific manufacturing procedure, and c. problems that occurred in design. 2) to determine the batch and quantity of defective products output by the step where the problem occurred in order to precisely work out the quantity of defective products: if a. the batch and quantity of the defective products made by one worker within the period of time T_worker is acquired according to the process_id of the worker; if $b$. the batch and quantity of the defective products manufactured within T_process based on the working procedure_id and T_processing; and if $\mathrm{c}$. the batch and quantity of the defective products designed is acquired per the designer_id and T_design. The query results need to meet the three constraints; that is, time, quantity and batch.

Time constraint: T_processing $=$ T_workers $=$ T_process is a time interval set by $T_{-}$part $\left(\mathrm{T}_{0} \ldots \mathrm{T}_{-}\right.$part... $\left.\mathrm{T}_{\mathrm{n}}\right) ; \mathrm{T}$-design $=\mathrm{T}$ _designer, the design batch and design date are determined according to the component_id.

Quantity constraint: Q_processing $\geq$ Q_process \&\& Q_design $\geq$ Q_designer \&\& Q_process $\geq$ Q_designer;

Batch constraint: B_process $\subseteq$ B_processing $\& \&$ BT_designer $\subseteq$ BT_design.

3) It is assumed that the tracing system uses distributed management among the supplier to shield the heterogeneity of the supplier distribution. Tracing is based on the batch list and the parent parts and completed in the superior supplier of supplier X, aiming to search the collection of subparts with defective parts and thereby target al the parent parts assembled with this subpart.

The specific procedures are as follows: 1) the order batch of the defective part 
or the subpart at the first layer is confirmed according to the batch list and order of the defective parts. 2) The dotted box in the middle denotes a cycle. For example, the supplier_id of the defective parts at the first layer is determined according to Figure 4. Step 3. (1), and the order batch of the defective subparts at the second layer is inquired through the purchasing information table. The rest was done in the same manner to conduct a looping execution of the process until the purchaser_id of the defective parts at the Mth layer is confirmed and when the order information of all defective parent parts is finally obtained, the cycle ends. 3) When the procurement information is collected according to the defective parent parts and the buyers, order batches, quantities and purchasing dates of all the parent parts are determined, the internal tracing ends.

\subsubsection{External Tracing System}

The internal tracing system aims at one auto supply chain while the external tracing system has to accommodate more than one auto sales chain. The manufacturing of auto parts belongs to mass customization. Therefore, the external tracing system should recall defective products based on the specific orders.

Figure 5 shows the external tracing system of defective vehicles. The specific procedures are as follows: 1) Based on the suppliers of the defective parent parts targeted by the internal tracing system, all the original equipment manufacturers purchasing the defective parent parts are traced. The order batch of the defective parent parts is acquired according to the purchasing information of the th auto manufacturer, and then the auto batches of all the defective parts are confirmed per information about the auto batches. 2) The Jth auto manufacturer traces all

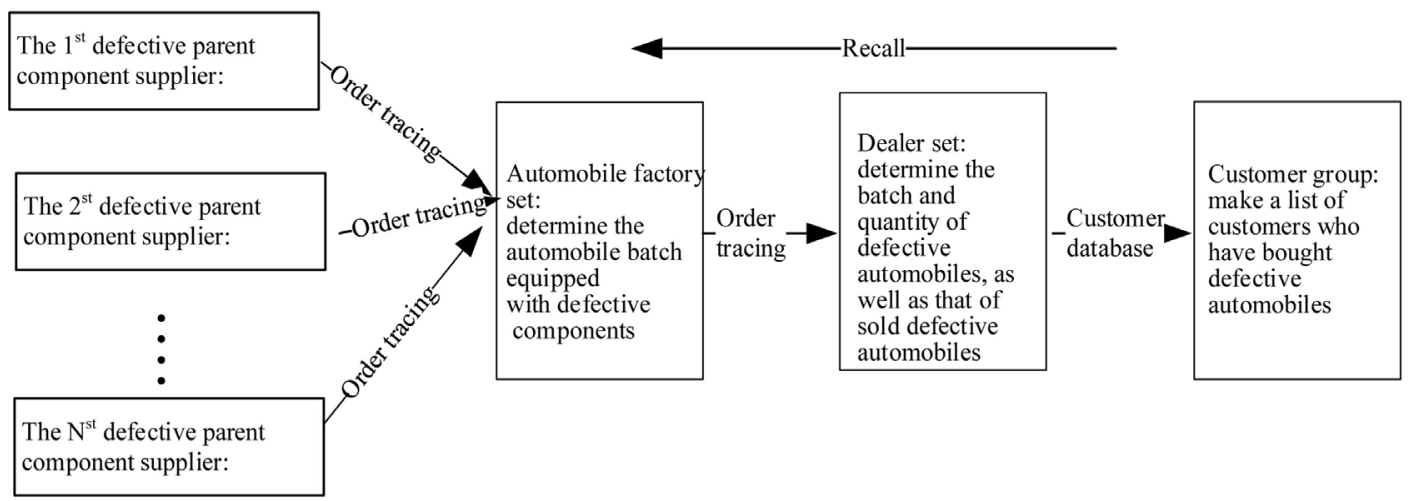

\begin{tabular}{|l|l|l|l|}
\hline $\begin{array}{l}\text { Order information of the } \mathrm{M}^{\text {th }} \\
\text { defective parent component/ } \\
\text { purchase information of the } \mathrm{J}^{\text {th }} \\
\text { automobile factory }\end{array}$ \\
\hline $\begin{array}{l}\text { Automobile factory -id } \\
\text { Automobile factory -name } \\
\text { Order batch } \\
\text { Order quantity } \\
\text { Inspector No. }\end{array}$ & $\begin{array}{l}\text { Automobile batch information } \\
\text { Order batch of defective components } \\
\text { Batch of automobiles equipped with } \\
\text { defective components } \\
\text { Production date }\end{array}$ & $\begin{array}{l}\text { Automobile batch } \\
\text { Pf the } \mathrm{J}^{\text {th }} \text { automobile factory }\end{array}$ \\
\hline $\begin{array}{l}\text { Order batch } \\
\text { Quantity of defective } \\
\text { automobiles } \\
\text { Batch of sold defective } \\
\text { automobiles } \\
\text { Quantity of sold defective } \\
\text { automobiles } \\
\text { Date }\end{array}$ \\
\hline
\end{tabular}

Figure 5. External tracing system. 
dealers that purchased the defective vehicles, and then determines the batch and quantity of defective vehicles as well as the quantity, batch and date of defective vehicles, which have already been sold per the purchasing information provided by the dealers. 3) After acquiring the contact information of clients who have purchased the defective vehicles according to their purchasing place and time, and the VIN codes of vehicles they purchased, the dealers recall the defective vehicles.

\subsection{Application of QR Code Technology in the Tracing System of Defective Products}

The QR code technology is mainly used for product identification and tracking, data collection, entry and information transmission in the tracing system. Detailed, comprehensive product data information and dynamic product tracking technology are the basis of an effective tracing system; the QR code identification principle can effectively meet the tracing system requirements for information collection and product tracking. The QR code embedded in the product can track products and record and transfer information about the key parts. Also, a waterproof, high temperature resistant, QR code can work in harsh environments.

Based on the node identification principle of the QR code sender, information needing to be identified is listed in Table 2 .

The production process is described below. The supplier provides the parts to the manufacturer who manufactures the parts. After a series of processes and with the participation of the designers and operators, the subparts are processed into parent parts, which are then assembled in the final working procedure. After that, the finished product enters the $4 \mathrm{~S}$ store to be purchased by one consumer, completing the normal direction flow from procurement to production, sales, and consumer. During such a process, information about the product, organizations and personnel involved above should be associated, as shown in Figure 6. The QR code associated with relevant information is printed onto the corresponding single items, cartons and vehicles. Table 3 shows the nodes needing to be recorded and their symbolic representation.

The above mechanism to associate information with nodes is realized by the linked list data structure in the system as shown in Figure 7.

Table 2. Encrypted and disclosed information coding of the QR code sender.

\begin{tabular}{lll}
\hline & $\begin{array}{l}\text { Identification code for suppliers } S^{i} \text {, identification code for } \\
\text { Encrypted } \\
\text { information }\end{array}$ & $\begin{array}{l}C^{i} \text {, identification code for the purchased parts } L^{i} \text {, identification } \\
\text { code for the improved parts } I^{i}, \text { identification code for new pars } \\
\text { QR code }\end{array}$ \\
conder & $A^{i}$, box code $B^{i}$, batch code $G^{i}$
\end{tabular}


Table 3. Symbols of the recorded nodes.

\begin{tabular}{ll}
\hline \multicolumn{1}{c}{ Nodes to be recorded } & \multicolumn{1}{c}{ Symbol } \\
\hline Supplier & $S^{i}$-Supply \\
Parts & $L^{i}$-Ling Bu Jian \\
Procedure & $P^{i}$-Procedure \\
Workers & $W^{i}$-Workers \\
Assembly units & $A^{i}$-Assembly \\
Box & $B^{i}$-Box \\
Group & $G^{i}$-Group \\
Vehicle & $V^{i}$-Vehicle \\
Consumer & $C^{i}$-Consumer \\
Improved parts & $I^{i}$-Improve \\
Manufacturing enterprise & $E^{i}$-Enterprise \\
\hline
\end{tabular}

The next step is the reverse tracing of the defective products. Upon receiving the vehicles to be repaired, the technicians detect the cause of the failure, finds the defective parts or components, and then scan the QR codes of those parts or components to easily confirm information about their supplier, production processes, operators, designers, batch number, vehicle, whether it was purchased by consumers, consumer information, and the $4 \mathrm{~S}$ store where it was sold. The detection personnel further identify the cause of the failure: Are the procured parts unqualified or is there any problem that occurred during the production process? For example, is this a design problem or a mistake caused by the operator? If the procured parts are unqualified, relevant information about the supplier can be acquired and the quality of parts purchased in the same batch should be checked to determine whether it is an individual case or the whole batch of parts are unqualified.

If there is a problem with the production process, whether there is a design problem or whether it is caused by improper operation of the operator should be checked. For whatever reason, it is important to identify the processes as well as personnel associated with it, and inspect the products manufactured in the same batch to determine whether they also have quality problems.

At this point, the causes and links of this defective product are found. Then, it is still necessary to determine whether it is an individual case or all the products in the same batch are unqualified. If all the products in the same batch are unqualified, then the models these products were assembled into need to be identified. Moreover, among these vehicles, those that have been sold and those in the warehouse need to be determined. Consumers who have purchased those vehicles need to be contacted to recall the vehicles, and those in the warehouse should be melted down and remade.

The above product process identification and defective product tracing avoids 
Node

Information association

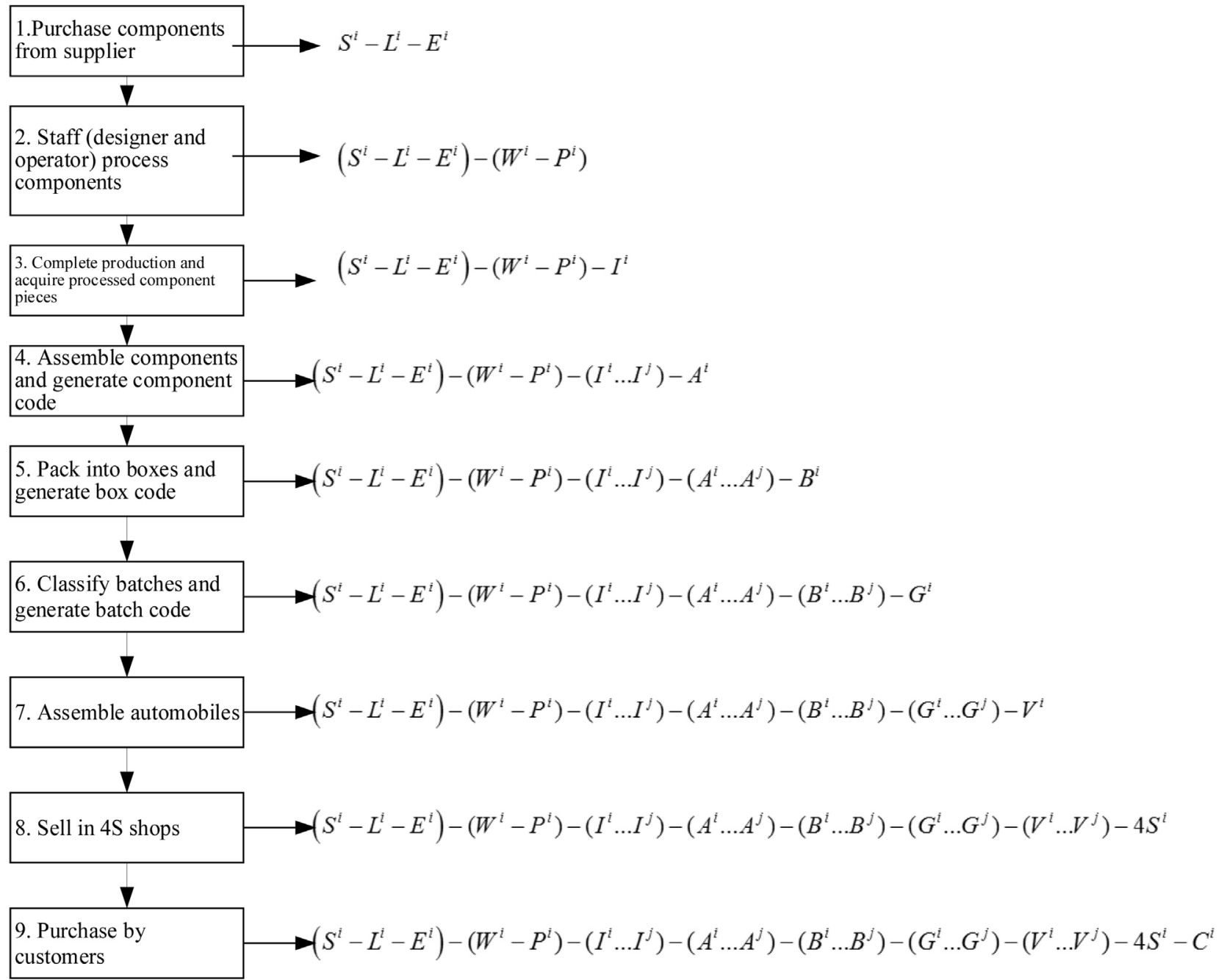

Figure 6. Node identification principle of product information diffusion.

the recall of qualified products, achieving a precise recall process and preventing the economic losses of enterprises.

\section{Conclusions and Future Research Work}

The novel contribution of the paper is that, based on the product information diffusion in consumer self-organized social networks, the control of the product information diffusion process was explored, making the following achievements and innovations. To solve the problem of node identification and information association in the process of information diffusion, a method to control the product information diffusion process was proposed based on the designed information identification principle of $\mathrm{QR}$ code senders, a method to create $\mathrm{QR}$ codes containing disclosed or encrypted information, and a method to generate the linked list associating information with nodes. This method addressed the threat of personal information leakage during user information transmission, 


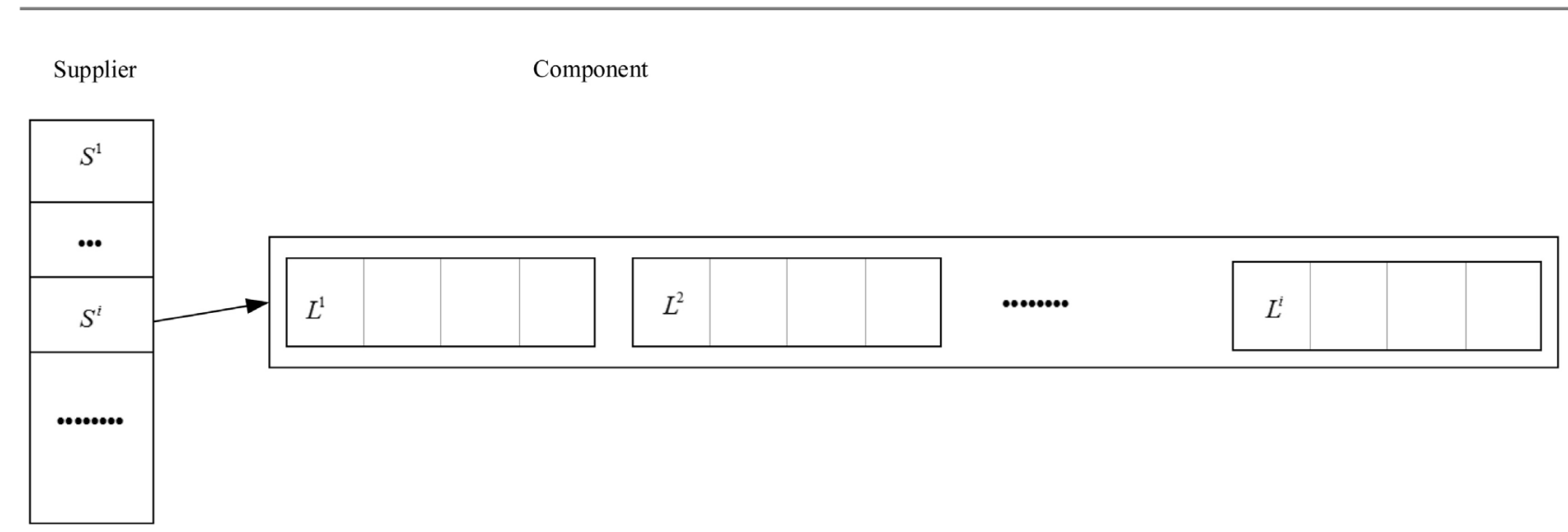

Process Staff on each process

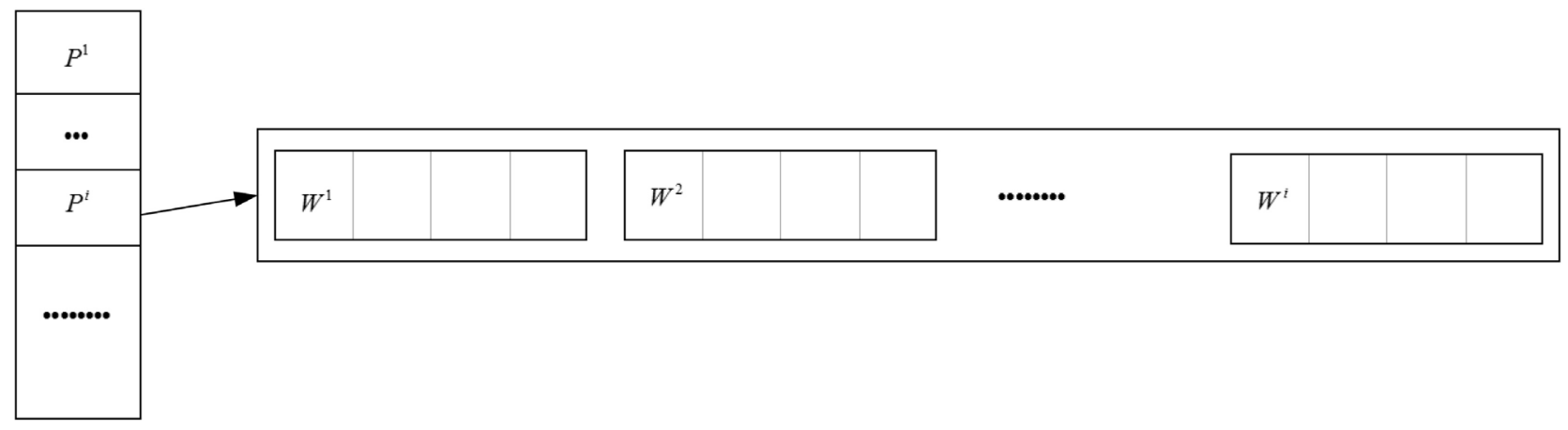

Single
product Information associated with single product

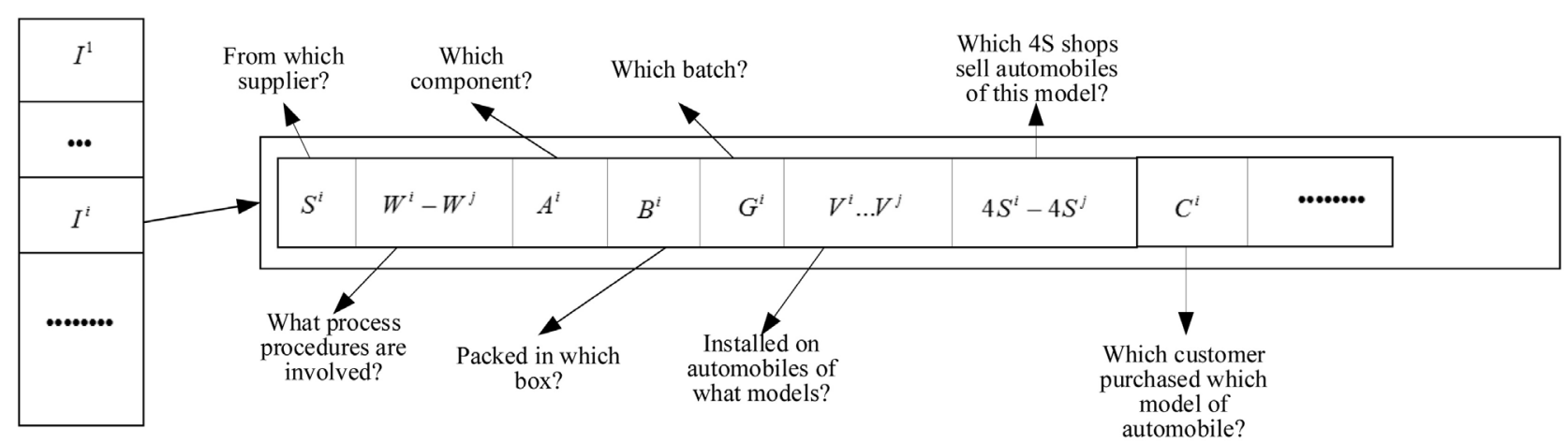

Figure 7. The linked list that associated information with specific nodes.

and achieved control of product information diffusion. Moreover, this method was applied to trace and record the technical process of defective products, realizing the accurate recall of defective vehicles.

In light of the findings of this paper, the following questions for future research are presented. As the diffusion of product information in social network is driven by spontaneous behavior of consumers, and the choice of consumers would be affected by many factors. And, the analysis of influencing factors and consumers' behavior selection also needs theoretical support from sociology, economics and psychology. Therefore, the simulation and control of product 
information diffusion could be carried out by multidisciplinary experiments.

\section{Acknowledgements}

This work is supported by Department of Education of Hebei Province (grant number QN2018259).

\section{Conflicts of Interest}

The authors declare no conflicts of interest regarding the publication of this paper.

\section{References}

[1] Cheng, J.J., Liu, Y., Shen, B., et al. (2013) An Epidemic Model of Rumor Diffusion in Online Social Networks. The European Physical Journal B, 86, 29. https://doi.org/10.1140/epjb/e2012-30483-5

[2] Dybiec, B. (2009) SIR Model of Epidemic Spread with Accumulated Exposure. The European Physical Journal B, 67, 377-383. https://doi.org/10.1140/epjb/e2008-00435-y

[3] Daley, D.J. and Kendal, D.G. (1964) Epidemic and Rumors. Nature, 204, 1118. https://doi.org/10.1038/2041118a0

[4] Daley, D.J. and Kendal, D.G. (1965) Stochastic Rumors. IMA Journal of Applied Mathematics, 1, 42-55. https://doi.org/10.1093/imamat/1.1.42

[5] Daley, D.J. and Gani (2000) Epidemic Modelling. Cambridge University Press, Cambridge.

[6] Tian, R.Y., Zhang, X.F. and Liu, Y.J. (2015) SSIC Model: A Multi-Layer Model for Intervention of Online Rumors Spreading. Physica A, 427, 181-191. https://doi.org/10.1016/j.physa.2015.02.008

[7] Denning, R.J. (1985) The Science of Computing: Super Networks. American Scientist, 73, 225-227.

[8] Xia, L.L., Jiang, G.P., Song, B. and Song, Y.R. (2015) Rumor Spreading Model Considering Hesitating Mechanism in Complex Social Networks. Physica A, 437, 295-303. https://doi.org/10.1016/j.physa.2015.05.113

[9] Wang, Y.Q., Yang, X.Y., Han, Y.L. and Wang, X.A. (2013) Rumor Spreading Model with Trust Mechanism in Complex Social Networks. Communications in Theoretical Physics, 59, 510-516. https://doi.org/10.1016/j.physa.2015.05.113

[10] Qian, Z., Tang, S.T., Zhang, X. and Zheng, Z.M. (2015) The Independent Spreaders Involved SIR Rumor Model in Complex Networks. Physica A, 429, 95-102. https://doi.org/10.1016/j.physa.2015.02.022

[11] Ma, J., Li, D.D. and Tian, Z.H. (2016) Rumor Spreading in Online Social Networks by Considering the Bipolar Social Reinforcement. Physica A, 447, 108-115. https://doi.org/10.1016/j.physa.2015.12.005

[12] Huo, L.A., Jiang, J.H., Gong, S.X. and He, B. (2016) Dynamical Behavior of Rumor Transmission Model with Holling-Type 2 Functional Response in Emergency Event. Physica A, 450, 228-240. https://doi.org/10.1016/j.physa.2015.12.143

[13] Giorno, V. and Spina, S. (2016) Rumor Spreading Models with Random Denials. Physica A, 461, 569-576. https://doi.org/10.1016/j.physa.2016.06.070 
[14] Huo, L.A., Wang, L., Song, N.X., et al. (2017) Rumor Spreading Model Considering the Activity of Spreaders in the Homogeneous Network. Physica A, 468, 855-865. https://doi.org/10.1016/j.physa.2016.11.039

[15] Zhu, L. and Wang, Y.G. (2017) Rumor Spreading Model with Noise Interference in Complex Social Networks. Physica A, 469, 750-760. https://doi.org/10.1016/j.physa.2016.11.119

[16] Liu, Y., Diao, S.M., Zhu, Y.X. and Liu, Q. (2016) SHIR Competitive Information Diffusion Model for Online Social Media. Physica A, 461, 543-553. https://doi.org/10.1016/j.physa.2016.06.080

[17] Kempe, D., Kleinberg, J. and Tardos, E. (2003) Maximizing the Spread of Influence through a Social Network. Proceedings of the 9th ACM SIGKDD International Conference on Knowledge Discovery and Data Mining, Washington DC, 24-27 August 2003, 137-146. https://doi.org/10.1145/956750.956769

[18] Clifford, P. and Sudbury, A. (1973) A Model for Spatial Conflict. Biometrika, 60, 581-588. https://doi.org/10.1093/biomet/60.3.581

[19] Chen, W., Collins, A., Cummings, R., et al. (2011) Influence Maximization in Social Networks When Negative Opinions May Emerge and Propagate. Proceedings of the 2011 SIAM International Conference on Data Mining, Mesa, 28-30 April 2011, 379-390. https://doi.org/10.1137/1.9781611972818.33

[20] He, X., Song, G., Chen, W. and Jiang, Q. (2012) Influence Blocking Maximization in Social Networks under the Competitive Linear Threshold Model. Proceedings of the 2012 SIAM International Conference on Data Mining, Anaheim, 26-28 April 2012, 463-474. https://doi.org/10.1137/1.9781611972825.40

[21] Li, Y., Chen, W., Wang, Y. and Zhang, Z.L. (2013) Influence Diffusion Dynamics and Influence Maximization in Social Networks with Friend and for Relationships. Proceedings of the 6th ACM International Conference on Web Search and Data Mining, Rome, 4-8 February 2013, 657-666. https://doi.org/10.1145/2433396.2433478

[22] Zhang, X., Zhu, J., Wang, Q., et al. (2013) Identifying Influential Nodes in Complex Networks with Community Structure. Knowledge-Based Systems, 42, 74-84. https://doi.org/10.1016/j.knosys.2013.01.017

[23] Chen, D., Lu, L., Shang, M.S., et al. (2012) Identifying Influential Nodes in Complex Networks. Physica A, 391, 1777-1787. https://doi.org/10.1016/j.physa.2011.09.017

[24] Kitsak, M., Gallos, L.K., Havlin, S., et al. (2010) Identification of Influential Spreaders in Complex Networks. Nature Physics, 6, 888-893.

https://doi.org/10.1038/nphys1746

[25] Kempe, D., Kleinberg, J. and Tardos, E. (2005) Influential Nodes in a Diffusion Model for Social Networks. Lecture Notes in Computer Science, 3580, 1127-1138. https://doi.org/10.1007/11523468_91

[26] Bae, J. and Kim, S. (2014) Identifying and Ranking Influential Spreaders in Complex Networks by Neighborhood Coreness. Physica A, 395, 549-559.

https://doi.org/10.1016/j.physa.2013.10.047 